\title{
Reconstructing The Moore-Gibson-Thompson Equation
}

https://doi.org/10.1515/msds-2020-0117

Received June 11, 2020; accepted November 26, 2020

Abstract: We are concerned with the inverse problem of recovering a third order Moore-Gibson-Thompson equation from a single observation of its solution at an arbitrary point. We show how to reconstruct its three unknown parameters and the memory kernel by using the Laplace transform.

Keywords: Moore-Gibson-Thompson equation, Inverse problem, Memory, Third order PDE

MSC: 35R30, 35Q70, 35L05, 35L35

\section{Introduction}

The objective of this note is to reconstruct the positive constants $\alpha, \beta, \gamma$ and the relaxation function $g$ of the following Moore-Gibson-Thompson (MGT) equation

$$
\begin{cases}u_{t t t}+\alpha u_{t t}-\beta \Delta u_{t}-\gamma \Delta u+\int_{0}^{t} g(t-s) \Delta u(s) d s=0, & \text { in } \Omega \times \mathbb{R}^{+}, \\ \partial_{v} u(x, t)=0, & \text { on } \partial \Omega \times \mathbb{R}^{+}, \\ u(x, 0)=u_{0}(x), \quad u_{t}(x, 0)=u_{1}(x), \quad u_{t t}(x, 0)=u_{2}(x) & \text { in } \Omega,\end{cases}
$$

from one observation of the solution at a single point, i.e. $u(b, t)$ for $t>0$. Here $\Omega \subset \mathbb{R}^{d}(d \geq 1)$ is a bounded domain with a smooth boundary, say $C^{2}$. The model in (1) describes the nonlinear propagation of acoustic waves (such as high frequency ultrasound waves) in a viscoelastic material, whose behavior and effect are governed by the convolution "memory" term $\int_{0}^{t} g(t-s) \Delta u(s) d s$. We refer to [6] for the derivation of the MGT equation and the list of references therein. These equations have been the focus of several recent research due to their wide range of applications in the medical and industrial fields of high intensity ultrasound waves.

The direct problem of (1) has received a lot attention. First Lasiecka and Wang established its wellposedness and showed exponential decay of the energy [6] and more general decay results followed, for example [7] for the subcritical case (i.e. $\alpha-\frac{\gamma}{\beta}>0$ ). These have been made more precise and optimal decay rates was obtained by Liu et al. [11]. For the critical case $\left(\alpha-\frac{\gamma}{\beta}=0\right)$ Dell'Oro et al. [4] proved a polynomial decay rate under more regular initial data.

The inverse problem for (1) with $g=0$ but $\alpha$ being a function of $x$ was treated by Liu and Triggiani $[9,10]$, who proved uniqueness of the coefficient $\alpha(x)-\frac{\gamma}{\beta}$ and also showed stability by Carleman estimates. However no reconstruction procedure were given. We recall that the well-posedness in this case was proven

Waled Al-Khulaifi: Department of Mathematics and Statistics, King Fahd University of Petroleum and Minerals, Dhahran 31261, Saudi Arabia, E-mail: waled.alkhulaifi@kfupm.edu.sa

${ }^{\star}$ Corresponding Author: Amin Boumenir: Department of Mathematics, UWG, Carrollton, GA 30118, USA, E-mail:

boumenir@westga.edu 
by Kaltenbacher et al. [5] and Marchand et al. [12].

Our aim in this note is to give an explicit reconstruction procedure of the coefficients $\alpha, \beta, \gamma$ and the kernel function $g$ found in (1) from a single observation of the solution at one point. To achieve this, we use the idea developed in [1], taking into consideration the nature of MGT equation under the subcritical case, and establish a simple computational method to reconstruct the sought parameters. Vespri et al. [3] considered a parabolic functional equation, where they showed that two measurements are enough to yield uniqueness.

\section{Preliminaries}

We now state sufficient assumptions that ensure global existence of the solution on (1).

$(A 1) \beta \alpha-\gamma>0$.

(A2)g: $\mathbb{R}^{+} \rightarrow \mathbb{R}^{+}$is a $C^{1}$ decreasing function satisfying

$$
0<g(0)<\alpha(\beta \alpha-\gamma), \quad \gamma-\int_{0}^{\infty} g(s) d s=\ell>0 .
$$

Proposition 1 ([6]). Assume that $\left(u_{0}, u_{1}, u_{2}\right) \in H^{1}(\Omega) \times H^{1}(\Omega) \times L^{2}(\Omega)$ and $(A 1)$ and $(A 2)$ are satisfied; then the problem (1) has a unique global (weak) solution

$$
u \in C^{1}\left(\mathbb{R}^{+} ; H^{1}(\Omega)\right) \cap C^{2}\left(\mathbb{R}^{+} ; L^{2}(\Omega)\right) .
$$

The fact that we have global existence of the solution, means that we can observe it over $(0, \infty)$, and so the use of the Laplace transform is possible to reconstruct the function $g$ over $(0, \infty)$. Observe that we only need to use the solution at one point only over the half line, i.e. $u(b, t)$ which provides an equivalent amount of data.

Now we can state the inverse problem:

Problem statement: Find initial functions $\left(u_{0}, u_{1}, u_{2}\right) \in H^{1}(\Omega) \times H^{1}(\Omega) \times L^{2}(\Omega)$, so to reconstruct the parameters $\{\alpha, \beta, \gamma\}$ and the memory function $g$ from a single observation of the solution at one point $\{u(b, t)\}_{t \geq 0}$ where $b \in \Omega$.

As we shall use pseudo-spectral methods associated the Neumann Laplacian, let us denote by $\lambda_{n}$ its eigenvalues and eigenfunctions by $\varphi_{n}$, i.e.

$$
\begin{cases}-\Delta \varphi_{n}(x)=\lambda_{n} \varphi_{n}(x), & \text { in } \Omega, \\ \partial_{\nu} \varphi_{n}(x)=0, & \text { on } \partial \Omega .\end{cases}
$$

where $\partial_{v}$ denotes the outward normal derivative. It is known that

$$
0=\lambda_{1}<\lambda_{2} \leq \lambda_{3} \leq \cdots \leq \lambda_{n} \leq \cdots,
$$

$\lim _{n \rightarrow \infty} \lambda_{n}=\infty$, and the set $\left\{\varphi_{n}\right\}_{n \geq 1}$, normalized by $\left\|\varphi_{n}\right\|=1$, is a basis for $L^{2}(\Omega)$.

As the unique global solution $u(., t) \in L^{2}(\Omega)$, means that it can be written as

$$
u(x, t)=\sum_{n=1}^{\infty} c_{n}(t) \varphi_{n}(x),
$$

where

$$
c_{n}(t)=\int_{\Omega} u(x, t) \varphi_{n}(x) d x
$$


The initial conditions translate into, for all $n \geq 1$,

$$
c_{n}^{(i)}(0)=\int_{\Omega} u_{i}(x, 0) \varphi_{n}(x) d x \quad \text { for } i=0,1,2 .
$$

Multiply (1) by $\varphi_{n}(x)$ and integrate over $\Omega$ to get

$$
\begin{aligned}
\int_{\Omega} u_{t t t}(x, t) \varphi_{n}(x) d x+\alpha \int_{\Omega} u_{t t}(x, t) \varphi_{n}(x) & d x-\beta \int_{\Omega} \varphi_{n}(x) \Delta u_{t}(x, t) d x \\
& -\gamma \int_{\Omega} \varphi_{n}(x) \Delta u(x, t) d x+\int_{0}^{t} g(t-s) \int_{\Omega} \varphi_{n}(x) \Delta u(x, s) d x d s=0 .
\end{aligned}
$$

which reduces to the functional equation, thanks to (3) and (5),

$$
c_{n}^{\prime \prime \prime}(t)+\alpha c_{n}^{\prime \prime}(t)+\beta \lambda_{n} c_{n}^{\prime}(t)+\gamma \lambda_{n} c_{n}(t)=\lambda_{n} \int_{0}^{t} g(t-s) c_{n}(s) d s
$$

with initial conditions

$$
c_{n}(0)=\int_{\Omega} u_{0}(x) \varphi_{n}(x) d x, \quad c_{n}^{\prime}(0)=\int_{\Omega} u_{1}(x) \varphi_{n}(x) d x, \quad c_{n}^{\prime \prime}(0)=\int_{\Omega} u_{2}(x) \varphi_{n}(x) d x .
$$

Since all sought parameters appear in (6), it is easy to extract some from it. For example since $\lambda_{1}=0$, we get from (6)

$$
c_{1}^{\prime \prime \prime}(t)+\alpha c_{1}^{\prime \prime}(t)=0 \quad \text { for all } t \geq 0
$$

as long as $c_{1}^{\prime \prime}$ is not trivial, i.e. $u_{2}$ is not orthogonal to $\varphi_{n}$. Hence by taking the limit, since the solution is $C^{3}[0, \infty)$.

$$
\alpha=-\frac{c_{1}^{\prime \prime \prime}(0)}{c_{1}^{\prime \prime}(0)} .
$$

We now show that a non trivial $c_{2}$ contains all the information required to reconstruct the sought coefficients. From (6) and by taking the limit as $t \rightarrow 0^{+}$, we deduce that $c_{2}$ satisfies,

$$
c_{2}^{\prime \prime \prime}(0)+\alpha c_{2}^{\prime \prime}(0)+\beta \lambda_{2} c_{2}^{\prime}(0)+\gamma \lambda_{2} c_{2}(0)=0 .
$$

Similarly differentiating (6)and taking the limit as $t \rightarrow 0^{+}$we deduce

$$
\begin{aligned}
\alpha c_{2}^{\prime \prime}(0)+\beta \lambda_{2} c_{2}^{\prime}(0)+\gamma \lambda_{2} c_{2}(0) & =-c_{2}^{\prime \prime}(0) \\
\alpha c_{2}^{\prime \prime \prime}(0)+\beta \lambda_{2} c_{2}^{\prime \prime}(0)+\gamma \lambda_{2} c_{2}^{\prime}(0) & =\lambda_{2} g(0) c_{2}(0)-c_{2}^{(4)}(0) \\
\alpha c_{2}^{(4)}(0)+\beta \lambda_{2} c_{2}^{\prime \prime \prime}(0)+\gamma \lambda_{2} c_{2}^{\prime \prime}(0) & =\lambda_{2}\left(g^{\prime}(0) c_{2}+g(0) c_{2}^{\prime}(0)\right)-c_{2}^{(5)}(0)
\end{aligned}
$$

By choosing

$$
c_{2}(0)=c_{2}^{\prime}(0)=0 \text { and } c_{2}^{\prime \prime}(0)=1,
$$

the above system reduces to a lower triangular matrix equation, which is easily solved by the Gaussian method

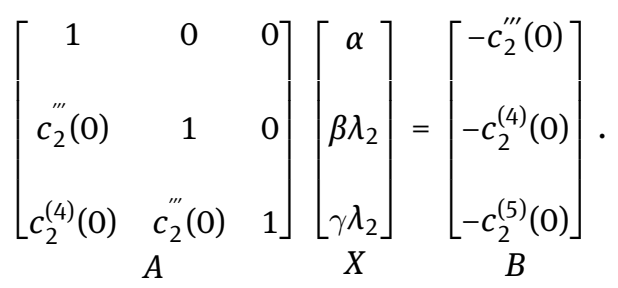


Since the determinant of A is 1 , the system above has the unique solution $\left(\alpha, \beta \lambda_{2}, \gamma \lambda_{2}\right)^{T}$.

Remark: The solution $u(x, t)$, is known to be globally $C^{2}$ in time, and so (1) implies that $u_{t t t}$ is also locally continuous it $t$. Thus its Fourier coefficients $c_{n}^{(3)}(t)$, are well defined and continuous in time. Therefore (6) holds in the classical sense and as the integral term is $C^{2}$, we obtain that $c_{n}^{(3)} \in C^{2}$, i.e. $c_{n} \in C^{5}$.

Once we have obtained the values $(\alpha, \beta, \gamma)$, we apply the Laplace transform, $\hat{g}(s)=\mathcal{L}(g)(s)$, to (6)

$$
\hat{c_{n}}(s)\left\{s^{3}+\alpha s^{2}+\beta \lambda_{n} s+\gamma \lambda_{n}\right\}=c_{n}^{\prime \prime}(0)+(s+\alpha) \hat{c}_{n}^{\prime}(0)+\left(s^{2}+\alpha s+\beta \lambda_{n}\right) c_{n}(0)+\lambda_{n} \hat{g}(s) \hat{c}_{n}(s),
$$

and so, for $n \geq 2$,

$$
\hat{g}(s)=\beta s+\gamma+\frac{1}{\lambda_{n}}\left(s^{3}+\alpha s^{2}\right)-\frac{1}{\lambda_{n} \hat{c}_{n}(s)}\left(c_{n}^{\prime \prime}(0)+(s+\alpha) c_{n}^{\prime}(0)+\left(s^{2}+\alpha s+\beta \lambda_{n}\right) c_{n}(0)\right)
$$

And if we use the boundary conditions in (7), the previous equation reduces to

$$
\hat{g}(s)=\beta s+\gamma+\frac{1}{\lambda_{2}}\left(s^{3}+\alpha s^{2}\right)-\frac{1}{\lambda_{2} \hat{c}_{2}(s)} .
$$

Note that it follows from condition $(A 2)$ that $g \in L^{2}(0, \infty)$, since

$$
\int_{0}^{\infty} g^{2}(t) d t \leq g(0) \int_{0}^{\infty} g(t) d t<\infty .
$$

Next recall that the Laplace transform is a bounded and invertible operator in $L^{2}(0, \infty)$, and so not only $\hat{g} \in L^{2}(0, \infty)$, it is also in the range of the Laplace transform, [2]. Thus its inverse Laplace transform exists and would yield back $g$.

It remains to see that we can observe $c_{2}$ from the solution $u(x, t)$, see (7), by choosing the initial condition as follows

$$
\begin{aligned}
u(x, 0) & =c_{2}(0) \varphi_{2}(x)=0 \\
u_{t}(x, 0) & =c_{2}^{\prime}(0) \varphi_{2}(x)=0 \\
u_{t t}(x, 0) & =c_{2}^{\prime \prime}(0) \varphi_{2}(x)=\varphi_{2}(x) .
\end{aligned}
$$

Then pick any point $b \in \Omega$ where $\varphi_{2}(b) \neq 0$ to read

$$
u(b, t)=c_{2}(t) \varphi_{2}(b)
$$

as all the $c_{n}=0$ if $n \neq 2$. Thus we proved the main result

Proposition 2. Taking $\left(u_{0}, u_{1}, u_{2}\right)=\left(0,0, \varphi_{2}\right)$ then we can reconstruct the parameters $\{\alpha, \beta, \gamma\}$ and the function $g$ using one observation only of the acoustic velocity potential $u$ at a single point $b \in \Omega$, such that $\varphi_{2}(b) \neq 0$.

Proof. With the given initial condition, the unique solution can be observed at $x=b$ is

$$
u(b, t)=c_{2}(t) \varphi_{2}(b)
$$

which exists for $t \geq 0$. It remains to avoid nodal curves, so the observation is not trivial.

Acknowledgments: The authors thank KFUPM for its continuous support. 


\section{References}

[1] A. Boumenir, The reconstruction of an equation of visco-elasticity, Nonautonomous Dynamical Systems, 5 , 1, 152-154 , 2018.

[2] A. Boumenir, and A. Al-Shuaibi, The inverse Laplace transform and analytic pseudo-differential operators J. Math. Anal. Appl. 228, 1, 16-36, 1998.

[3] F. Colombo, D. Guidetti, and V. Vespri, Some global in time results for integro-differential parabolic inverse problems, Lecture Notes in Pure and Applied Mathematics, 251, 35-58, 2006, 1999.

[4] F. Dell'Oro, and I. Lasiecka, and V. Pata, The Moore-Gibson-Thompson equation with memory in the critical case, Journal of Differential Equations, 261, 7 , 4188-4222 , 2016.

[5] B. Kaltenbacher, and I. Lasiecka, and R. Marchand, Well-posedness and exponential decay rates for the Moore-GibsonThompson equation arising in high intensity ultrasound, Control and Cybernetics, 40, 971-988, 2011

[6] I. Lasiecka, and X. Wang, Moore-Gibson-Thompson equation with memory, part I: exponential decay of energy, Zeitschrift fur angewandte Mathematik und Physik, 67, 2-17, 2016

[7] I. Lasiecka, and X. Wang, Moore-Gibson-Thompson equation with memory, part II: General decay of energy, Journal of Differential Equations, 259,12 , 7610-7635 , 2015

[8] I. Lasiecka, Global solvability of Moore-Gibson-Thompson equation with memory arising in nonlinear acoustics, Journal of Evolution Equations, 17 , 1, 411-441, 2017

[9] S. Liu, and R. Triggiani, Inverse problem for a linearized Jordan-Moore-Gibson-Thompson equation, New Prospects in Direct, Inverse and Control Problems for Evolution Equations, 305-351, 2014.

[10] S. Liu, and R. Triggiani, An inverse problem for a third order PDE arising in high-intensity ultrasound: Global uniqueness and stability by one boundary measurement Journal of Inverse and Ill-Posed Problems, 21, 6, 825-869, 2013.

[11] W. Liu, Z. Chen, and D. Chen, New general decay results for a Moore-Gibson-Thompson equation with memory Applicable Analysis , 1-19, 2019,

[12] R. Marchand, T. McDevitt, and R. Triggiani, An abstract semigroup approach to the third-order Moore-Gibson-Thompson partial differential equation arising in high-intensity ultrasound: structural decomposition, spectral analysis, exponential stability, Mathematical Methods in the Applied Sciences, 35 , 15 , 1896-1929, 2012. 